\title{
Charting Molecular Landscapes Using Cryo-Electron Tomography
}

\author{
Laura Burbaum, ${ }^{1,2}$ Miroslava Schaffer, ${ }^{1 *}$ Benjamin D. Engel, ${ }^{1}$ Julia Mahamid, ${ }^{1}$ \\ Sahradha Albert, ${ }^{1}$ Radostin Danev, ${ }^{1}$ Wolfgang Baumeister, ${ }^{1}$ and Jürgen M. Plitzko ${ }^{1}$ \\ ${ }^{1}$ Max Planck Institute of Biochemistry, Department of Molecular Structural Biology, Am Klopferspitz 18, \\ 82152 Martinsried, Germany \\ ${ }^{2}$ Max Planck Institute of Biochemistry, Department of Cellular and Molecular Biophysics, Am Klopferspitz 18, \\ 82152 Martinsried, Germany
}

*schaffer@biochem.mpg.de

\begin{abstract}
Cryo-electron tomography (CET) can visualize protein complexes within cellular volumes with molecular resolution. However, most cells are too thick to be imaged whole by this technique. Thinning cells with a focused ion beam (FIB) produces ideal samples for CET, opening windows into the native cellular environment. FIB milling is more efficient and less susceptible to artifacts than cryo-sectioning with a diamond knife, providing an elegant approach for thinning frozen biological material. This new advance in sample preparation has enabled the first high-resolution CET studies of macromolecules in situ.
\end{abstract}

\section{Introduction}

For centuries, humanity has been fascinated by the possibility of seeing beyond the limitations of human vision. The development of the light microscope was the starting point for investigations of objects that are too small to be seen by the naked eye. Over many generations of researchers, the resolution limit was pushed to smaller and smaller values. Super-resolution light microcopy techniques can now reach a resolution from up to $10 \mathrm{~nm}$, determining the positions of single fluorescent molecules inside cells with high precision. Nevertheless, light microscopy doesn't provide information about the structural nature of cellular components. On the other hand, $\mathrm{x}$-ray crystallography and cryo-electron microscopy (cryo-EM) have revealed the structures of several thousand biological complexes. The flood of three-dimensional protein structures has massively expanded our biological understanding of the inner machinery of the cell.

Most of these structures, however, were obtained from isolated proteins and organelles. As such, they cannot tell us how a protein complex behaves in its physiological environment, as part of the crowded reaction network in the cell. Until recently, it was not possible to take high-resolution pictures of the cellular interior. One reason for this is the limitation of transmission electron microscopy (TEM), where resolution is severely deteriorated for samples thicker than $300 \mathrm{~nm}$. This is primarily a result of inelastic electron scattering events that occur when electrons pass through the sample. In thick samples, this effect is stronger, causing dark, blurry images. Filtering of inelastic scattered electrons can diminish, but not completely compensate, for this effect.

Nonetheless, the first high-resolution images of the interior of the cell were recently published. These three-dimensional views revealed novel protein arrays within the Golgi cisternae [1], shed light on the structures of individual nuclear pore complexes [2], and visualized polyribosomes attached to the endoplasmic reticulum [3]. All of these macromolecular complexes were captured in their native environment using cryo-electron tomography (CET). How was that possible?

The idea is not new. Since cryo-EM is limited by the thickness of the sample, material that is too thick must be cut into thinner slices for optimal image acquisition. For decades, researchers have used diamond knives to cut cellular material. However, this technique has a low throughput, and specimens suffer from disturbing artifacts like curved sections, crevasses introduced by the knife, and compression in the cutting direction. These artifacts limited the obtainable image quality, hindering data analysis and evaluation.

In the aforementioned high-resolution studies [1-3], researchers used a focused ion beam (FIB) instead of a diamond knife to cut the samples. The FIB was initially developed for material science applications, but over the last several years has found its way into biological research. Most commonly, gallium ions are used for the precise milling of different kinds of material. Using a dual-beam FIB instrument equipped with an ion beam, an electron beam column, and a cryo-stage, frozen hydrated biological material can be thinned to any desired thickness [4].

The FIB literally opens a window into the cell, through which the cellular interior can be imaged in three dimensions using CET. The combination of FIB with CET now clears the way for achieving what many microscopists have long desired: snapshots of the molecular landscape of the cell.

\section{Materials and Methods}

To capture the native cellular environment, samples are not stained but rather are rapidly frozen directly on the EM grid, a small carrier composed of a fine mesh coated with a thin layer of perforated carbon foil. The grid carrying the cells is plunged with a guillotine-like device into liquid ethane. Due to the high freezing speed, ice crystal formation is avoided. The water in the sample is instead fixed in a vitreous state, in which cellular structures are preserved. From this point on, the sample must be kept at cryogenic conditions all times-including transfer between instruments and during data acquisition-to prevent devitrification (because ice crystal formation destroys the biological structures). To simplify the handling of the sample during the next steps, the frozen EM grids are loaded into Autogrid supports, which serve as sturdy frames around the grids to stabilize the fragile biological specimens. Before transferring a grid to the FIB for milling, cellular areas of interest can be identified with the help of correlated 


\section{IN SITU MADE SIMPLE}

In situ microscopy just got a lot easier. Meticulously crafted by our world-class engineers, the next-generation Protochips holders deliver heating and electrical biasing in liquid or gas to your electron microscope with the click of a button. Self aligning parts and intuitive software controls mean you can focus on what matters, your experiment. Find out more at: 
three-dimensional fluorescence microscopy at cryogenic conditions. This information can later be used to target specific features for FIB milling.

Grids are placed into a shuttle, which is then transferred onto a cryostage inside the FIB. The actual milling with the ion beam requires several additional preparation steps [5]. To improve the milling process and the quality of the lamellas, two thin layers of platinum are applied. Within the FIB cryo-prep chamber, an initial 5 to $20 \mathrm{~nm}$ thick platinum layer is sputtered over the whole grid to reduce the charging of the specimen. This layer not only minimizes charging effects during imaging, thus improving the detection of the cellular features with the scanning electron microscope (SEM), but it also reduces charge-induced drift during lamella preparation, thus increasing milling precision. Additionally, an approximately $2 \mu \mathrm{m}$ thick organometallic platinum layer is deposited using a gas injection system (GIS) to protect the sample surface during milling (Figure 1a). This dense layer minimizes curtaining-non-uniform ion beam milling rates due to the variable density of the sample. After application of these two different platinum layers, the sample is milled with the ion beam.

To mill sections that are almost parallel to the grid surface, the FIB shuttle is pre-tilted with a shallow angle between the sample surface and the ion beam. Gallium ions are directed in targeted patterns across the sample surface, removing material from the specimen in a stepwise manner. The user can monitor the milling process with the SEM to control the quality of the lamella and its thickness. The
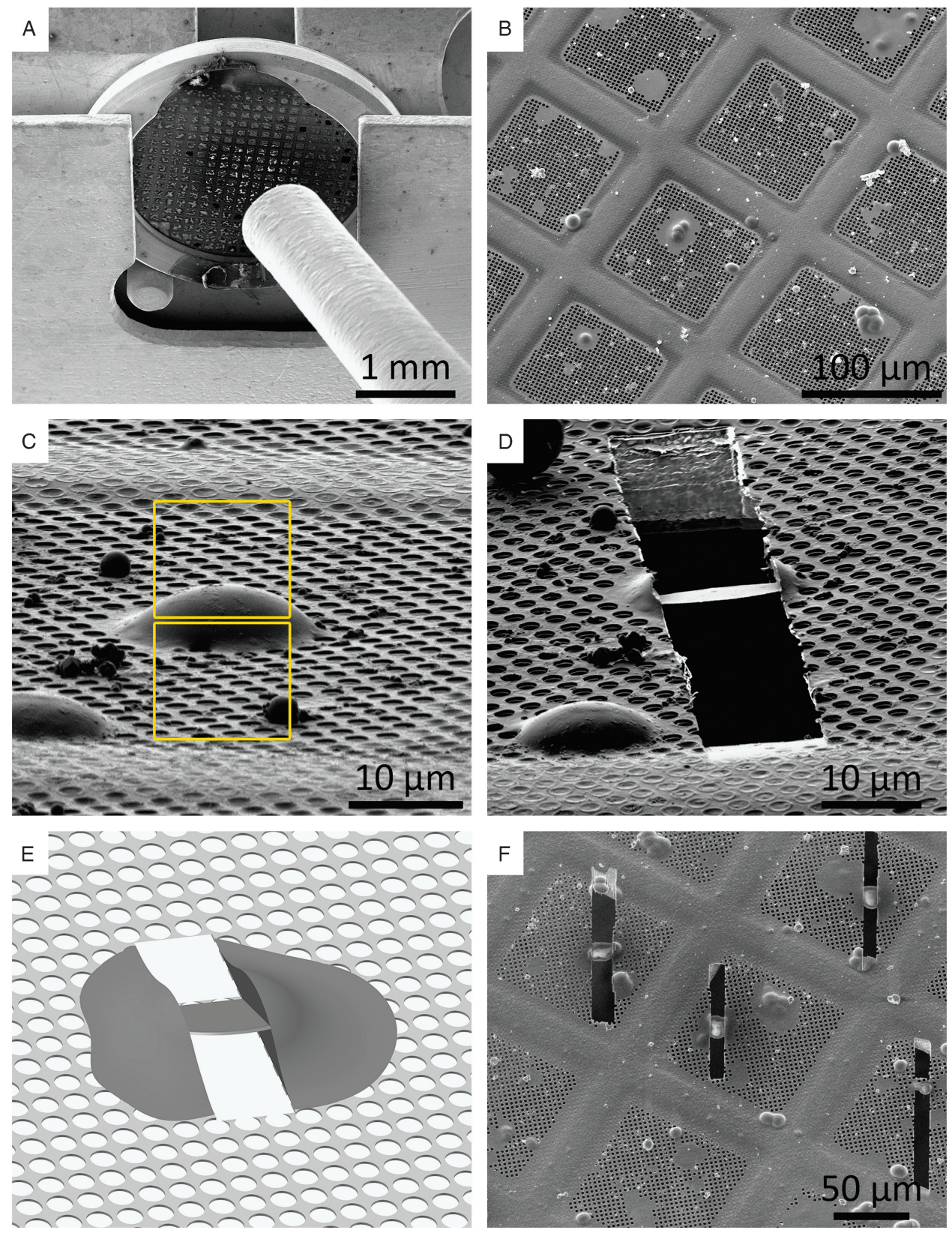

Figure 1: Example cryo-FIB-prepared lamella of Chlamydomonas alga cell. (A) SEM image of an AutoGrid while the GIS needle is inserted for the organometallic Pt deposition. SEM image (B) and FIB-induced secondary electron image (C) of a TEM grid with frozen Chlamydomonas cells. Two rectangle patterns indicate the areas where the material is cut to create a lamella. (D) FIB-induced secondary electron image of the final thinned lamella. (E) Schematic of a finished lamella. The direction of FIB milling is from left to right. (F) SEM image of a TEM grid with four prepared lamellas.

lamella is normally generated by cutting areas above and below the region of interest (Figure $1 b-d$ ). At a specific tilt angle $\left(3^{\circ}-18^{\circ}\right.$ specimen tilt), two rectangular patterns on each side of the desired lamella are milled simultaneously, while their distance to one another is decreased stepwise to thin the slice. Material is initially removed at high ion beam current (100-500 pA), whereas the final cleaning steps are performed at beam currents of 30-50 pA for more precise milling. The finished lamella (Figure 1d, 1e) doesn't have direct grid contact but is stabilized by the surrounding uncut biological material and ice. Ideally, the lamella should have a uniform thickness

of $60-300 \mathrm{~nm}$ throughout the milled region. After milling, the lamella is coated with a final thin layer of sputtered platinum, as the biological material is not sufficiently conductive for CET investigations. This layer must continuously and uniformly cover the surface of the new lamella but should be thin enough $(<5 \mathrm{~nm})$ to not impair the contrast of the sample during CET.

Even though many accurate steps are required for the successful preparation of one lamella, an experienced user can mill 4-10 lamellas on one EM grid during an eight-hour session (Figure 1e). Each lamella can span a surface of up to $600 \mu \mathrm{m}^{2}$, 


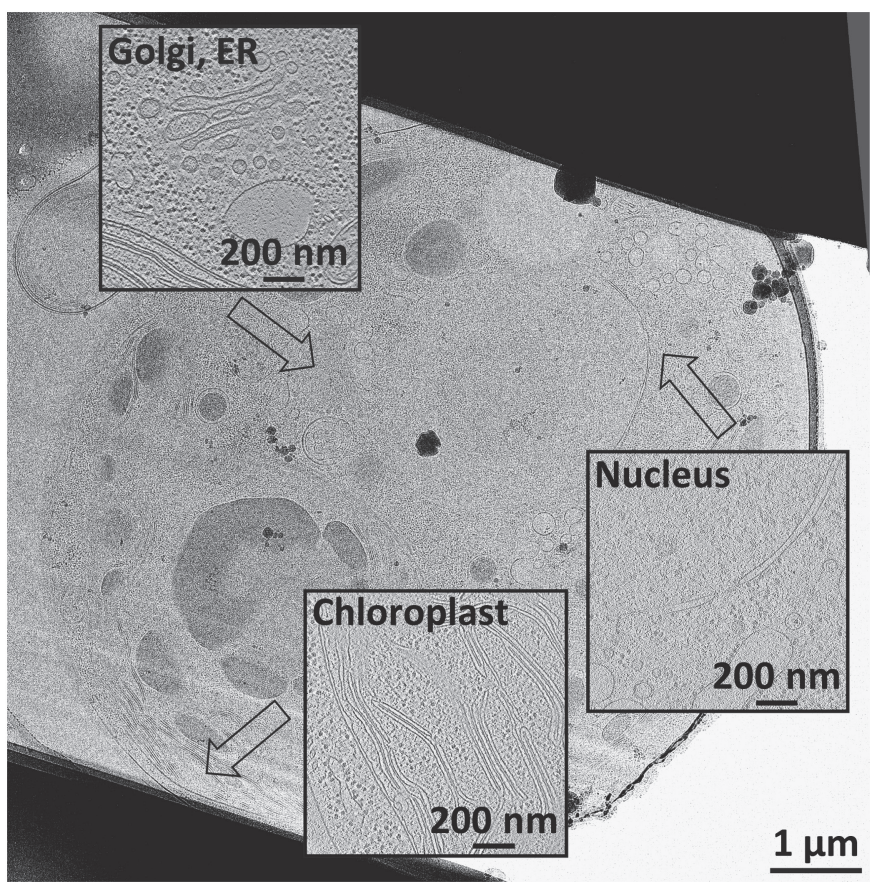

Figure 2: Cryo-EM/ET on a FIB-milled lamella prepared from a Chlamydomonas alga cell, supported by non-ablated cell material on each side. Different organelles of the Chlamydomonas cell are highlighted by arrows, and slices from tomographic volumes depicting these organelles are shown in the insets.

allowing the acquisition of several tomograms within the same lamella.

After successful preparation of the lamellas, the grids are removed from the FIB and transferred to the TEM. Here, tomographic data can be acquired using both zero-loss energy filtering and Volta phase plate imaging to allow direct electron detection at close to zero defocus. Tomograms are acquired to reveal three-dimensional views of the pleomorphic structures inside the cell; the area of interest is imaged multiple times at different angular orientations to produce a tilt-series [6]. The single pictures in the tilt-series are later computationally aligned and reconstructed to generate a three-dimensional representation of the cellular environment. To minimize radiation damage to the sample, the total electron dose is partitioned over the whole tilt-series, yielding a low signal-tonoise ratio for the single images. Fortunately, recent advances in instrumentation have improved CET significantly. With new direct electron detector cameras, electrons are measured directly without having to be first converted into photons, thus providing sub-nanometer resolutions for CET. Phase plates, and especially the thin Volta phase plate [7], improve the contrast of images of vitrified samples, enhancing the signal-tonoise ratio at low frequencies and allowing imaging at close to zero defocus.

\section{Results}

The combination of FIB, CET, and the Volta phase plate enables the acquisition of high-resolution snapshots of the cellular interior, in which different organelles including the Golgi, endoplasmic reticulum, nucleus, and chloroplast, can be easily identified (Figure 2). Large macromolecules such as ribosomes and ordered helical protein structures like actin filaments are also observable within the crowded cytoplasm.

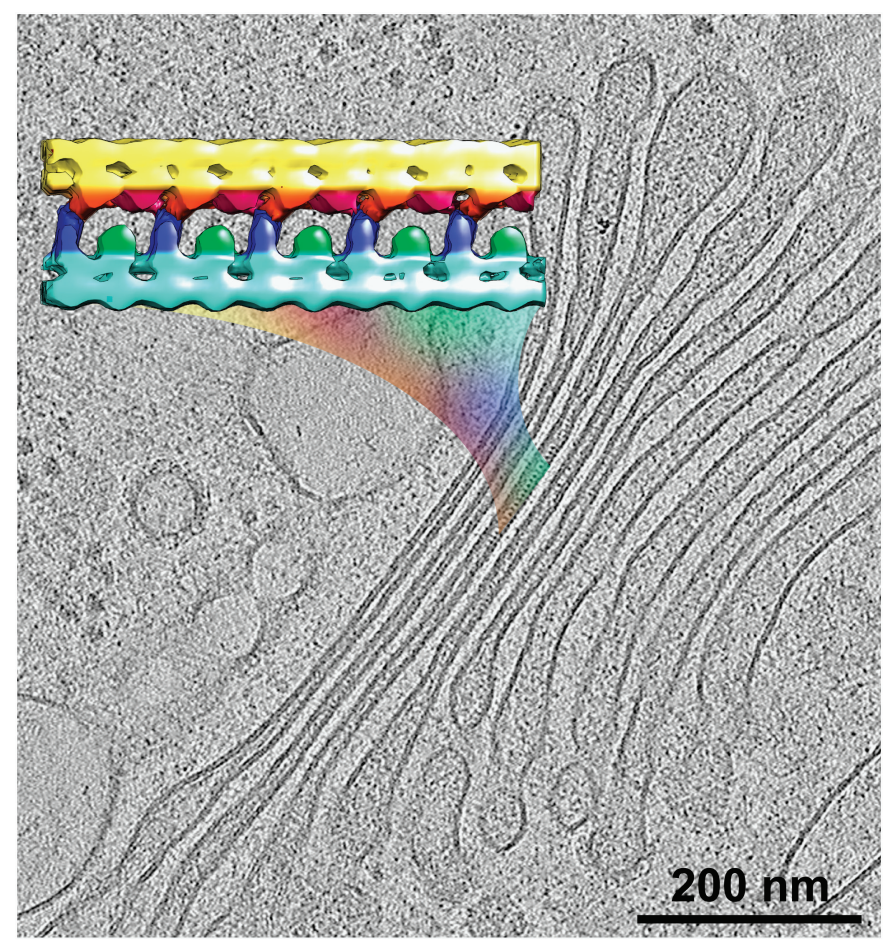

Figure 3: Example slice from a tomographic volume showing an extended array of luminal projections within a cisterna of the trans-Golgi. The colored inset displays a molecular structure derived from the data, revealing the different components of the protein array. The cis-side membrane and its embedded proteins are depicted in cyan, and the trans-side membrane and proteins are shown in yellow.

The quality of the tomograms even allows for structural analysis of single protein complexes in situ. To illustrate the potential of this method to reveal the molecular organization of cells, we review the following results from two studies that used FIB and CET to study the architecture of the cellular environment in different organisms.

New structure inside Golgi cisternae. High-resolution views of the native cellular environment not only provide new insights into known macromolecules, but sometimes also uncover structures that have never been identified before. B. Engel et al. [1] discovered a novel protein array within the narrow Golgi cisternae by carefully examining tomograms of the green alga Chlamydomonas (Figure 3). Subtomogram averaging revealed the molecular structure of an asymmetric membrane zipper formed by luminal projections that span the cisternae, providing insights into the mechanisms that shape Golgi architecture and orchestrate cargo transport through the organelle.

HeLa human cancer cells. J. Mahamid et al. [2] used the combination of FIB and CET to shed light on the nuclear periphery of HeLa human cancer cells (Figure 4). Their data gave the first insights into the organization of actin, microtubules, polyribosomes, and intermediate filaments in the vicinity of the nuclear envelope. The quality of the data enabled not only the detection of individual protein complexes within the crowded environment, but also the observation of structural changes on a single complex level. Cross sections of the nuclear pore complexes embedded in the nuclear envelope revealed structural details including the eight protomers and 
the cytoplasmic ring. This study impressively shows that structural variations can be identified for single complexes within the cellular environment, without the need for computationally averaging thousands of macromolecules.

\section{Conclusion}

In the past, CET was limited by specimen thickness. High-fidelity tomograms of the cellular interior were nearly impossible to obtain because the only available method for thinning cells, cryo-sectioning with diamond knives, distorted the sample with compression and crevasse artifacts. The FIB provides a solution for cellular CET by milling biological material under cryogenic conditions to produce thin slices of cellular material that are free of such artifacts. Thus, a new range of investigations has become possible for the first time.

The recent technical advancements in FIB and CET enable in situ imaging of native complexes with molecular resolution, thus bridging the gap between light microscopy and atomic-resolution techniques like single-particle electron microscopy. The synergistic combination of FIB and
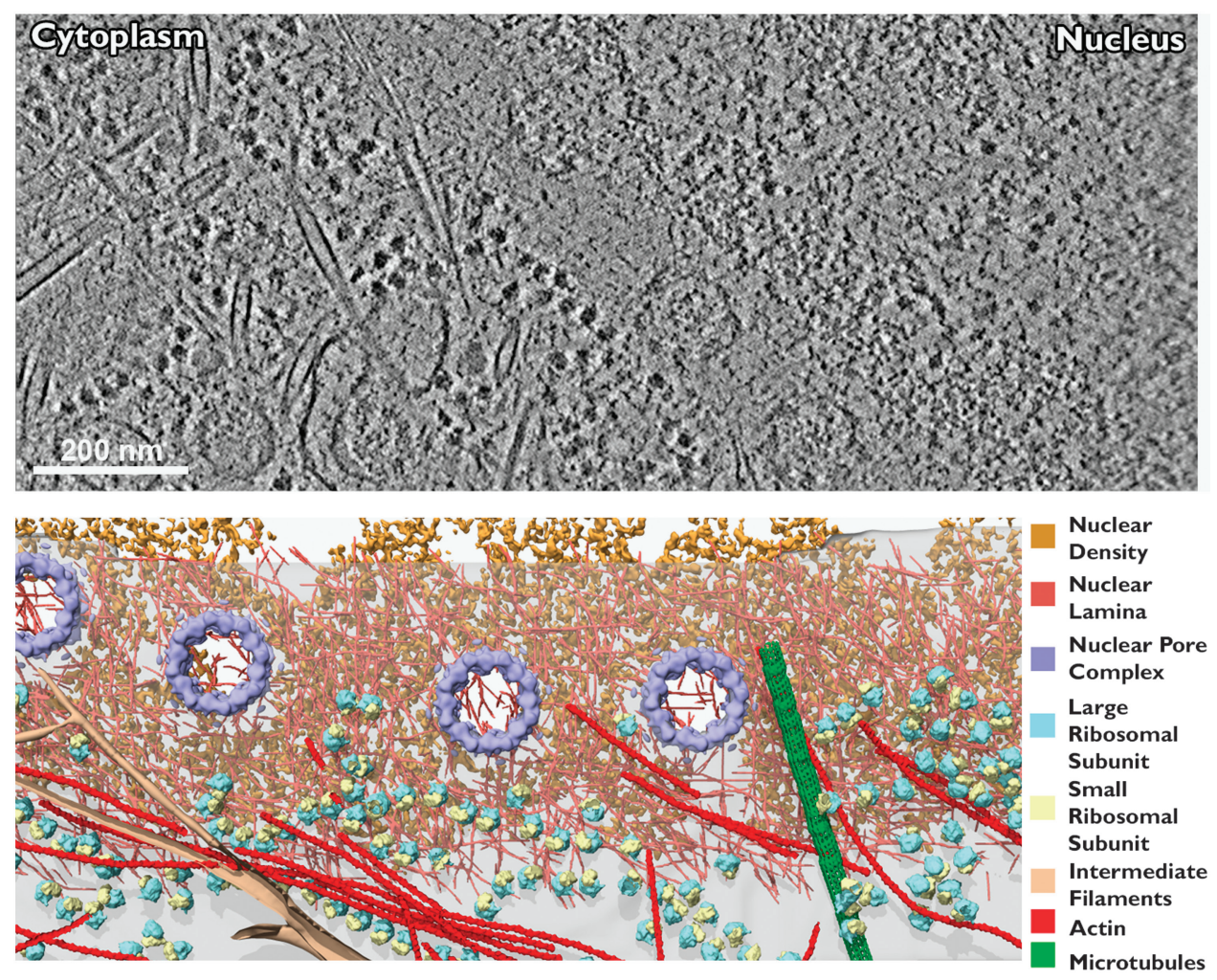

Figure 4: Example tomographic slice and segmented view of the vicinity of the nuclear envelope in a HeLa cell. The high contrast of the phase plate data allows for direct visualization and identification of different molecular complexes in the nuclear periphery and nuclear envelope, such as ribosomes, actin filaments, and nuclear pore complexes.

\section{PELCO Silicon Nitride}

\section{\& Silicon Dioxide Membranes}

\section{Next Generation SiN TEM Support Films}

- Robust and clean 8, 15, 50 and $200 \mathrm{~nm}$ SiN substrates

- $ø 3.0 \mathrm{~mm}$ frame

- EasyGrip ${ }^{\mathrm{T}}$ edges

- Free from debris

- Super flat 8, 15, and 40nm silicon dioxide substrates

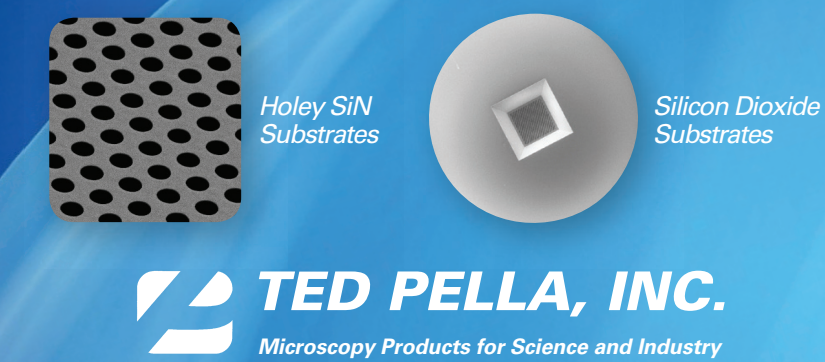

www.tedpella.com sales@tedpella.com 800.237.3526
CET holds promise for multi-scale cellular imaging, combining the structural information of macromolecular complexes with information about their location within the cell. Decades after the development of electron microscopy, technical improvements and hybrid approaches have allowed CET to become a key technique for exploring the native cellular environment.

\section{Acknowledgements}

We are grateful to the department's workshop for the design and production of various tools. M.S. was supported by the European Commission grant agreement ERC-2012-SyG_318987ToPAG. J.M. was supported by postdoctoral research fellowships from EMBO and HFSP, and by the Weizmann Institute Women in Science Program. B.E. was supported by a postdoctoral research fellowship from the Alexander von Humboldt Foundation. We also acknowledge support from the Deutsche Forschungsgemeinschaft Excellence Clusters CIPSM and SFB 1035, FEI Company, and the Max Planck Society.

\section{References}

[1] BD Engel et al., Proc Natl Acad Sci USA 112(36) (2015) 11264-69.

[2] J Mahamid et al., Science 351(6276) (2016) 969-72.

[3] S Pfeffer et al., Nat Commun 8 (2017) 14516.

[4] E Villa et al., Curr Opin Struct Biol 23(5) (2013) 771-77.

[5] M Schaffer et al., J Struct Biol 197(2) (2017) 73-82.

[6] V Lucić et al., Annu Rev Biochem 74 (2005) 833-65.

[7] R Danev et al., Proc Natl Acad Sci USA 111(44) (2014) 15635-40. 


\section{The Swiss Army Knife of Microscopy}

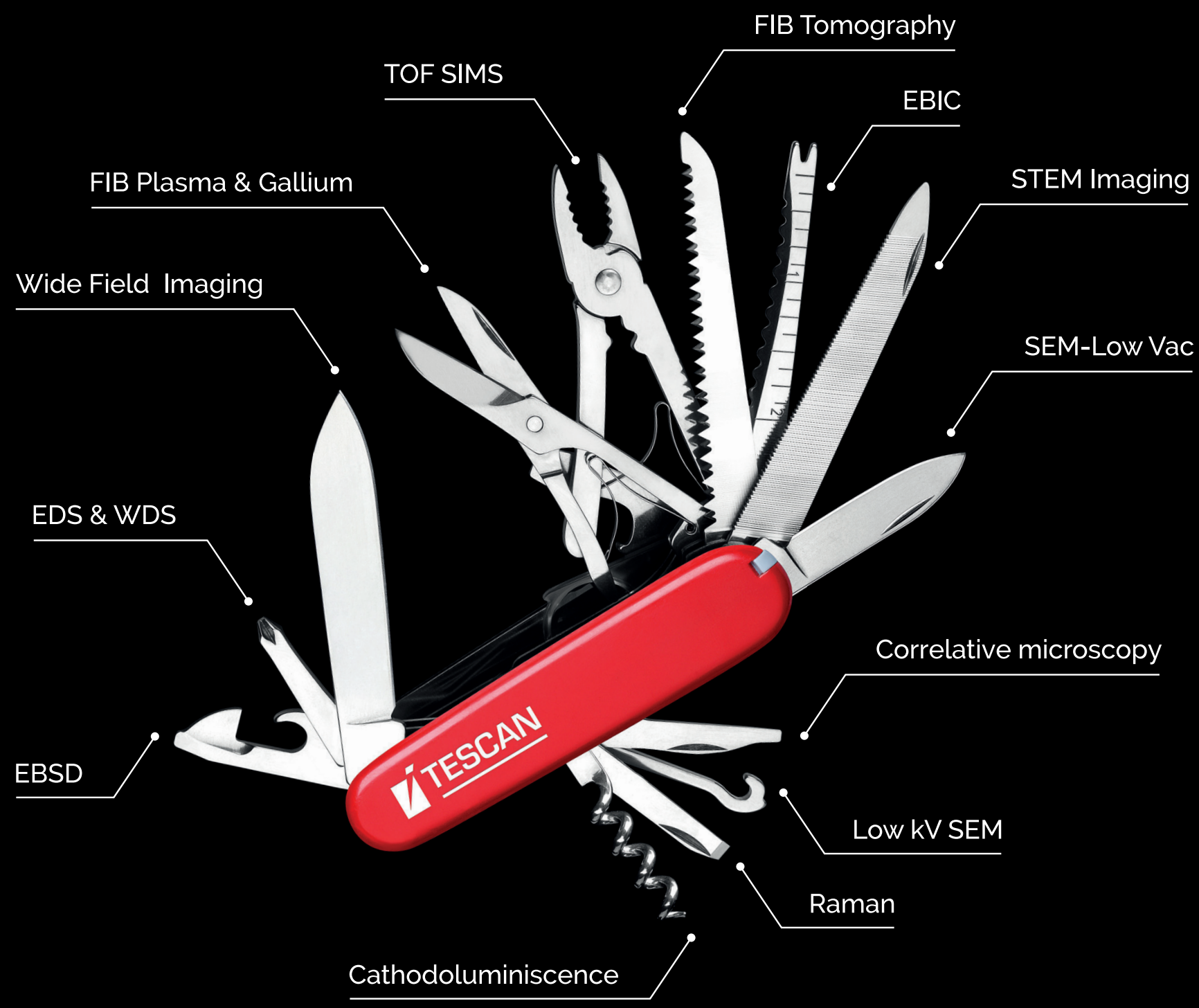

\section{Always have the right tool on hand!}

